\title{
Phase Closure Nulling: results from the 2009 campaign
}

\author{
Gilles Duvert ${ }^{a}$, Fabien Malbet ${ }^{a}$, Alain Chelli $^{a}$, Rafael Millan-Gabet ${ }^{b}$, \\ John D. Monnier ${ }^{c}$, Gail H. Schaefer ${ }^{d}$ \\ ${ }^{a}$ Lab. d'Astrophysique de Grenoble (LAOG), UMR 5571 Univ. J. Fourier/CNRS, BP 53, \\ F-38051 Grenoble cedex 9, France; \\ ${ }^{b}$ California Institute of Technology, NExScI, MC 100-22, Pasadena CA 91125, USA; \\ ${ }^{c}$ Univ. of Michigan, 941 Dennison Building, 500 Church Street, Ann Arbor, MI 48109, USA; \\ ${ }^{d}$ CHARA Array of Georgia State University, Mount Wilson Observatory, Mount Wilson, CA \\ 91023, USA.
}

\begin{abstract}
We present here a new observational technique, Phase Closure Nulling (PCN), which has the potential to obtain very high contrast detection and spectroscopy of faint companions to bright stars. PCN consists in measuring closure phases of fully resolved objects with a baseline triplet where one of the baselines crosses a null of the object visibility function. For scenes dominated by the presence of a stellar disk, the correlated flux of the star around nulls is essentially canceled out, and in these regions the signature of fainter, unresolved, scene object(s) dominates the imaginary part of the visibility in particular the closure phase. We present here the basics of the PCN method, the initial proof-of-concept observation, the envisioned science cases and report about the first observing campaign made on VLTI/AMBER and CHARA/MIRC using this technique.
\end{abstract}

Keywords: Observing Technique, Optical Interferometry, Complex Visibility, Closure Phase, Spectroscopic Binaries, Companions

\section{INTRODUCTION}

Phase Closure Nulling (hereafter PCN) was introduced in a paper by Chelli et al. (2009). ${ }^{1}$ In that reference, the authors did not emphasize the very peculiar series of coincidences that lead to the technique described here, which in retrospect merit to be told.

It really all stems from a few minutes of observations taken in February 2008 during a night of an AMBER Task Force commissioning run of. ${ }^{2}$ The purpose of this commissioning run was to find, and if possible to cure, some obvious instabilities in the transfer function of AMBER that rendered the absolute calibration of the visibilities very difficult. We found in particular that the entrance polarizers (one per beam) were the cause of a variable fringe beating which biased the visibilities. We thus removed the polarizers to test on a series of calibrators that the stability of the instrument was indeed improved. We chose a series of calibrators from the ESO calibrator list, located at various positions in the sky and also covering a large range of fluxes. Among these calibrators, the brightest was the K5III star $\sigma$ Puppis.

This night run was successful, and demonstrated that the stability of the instrument was greatly improved without the polarizers. Then, the polarizers were put back, since otherwise the instrument would not have been, in ESO terms, nominal. Finally, the culprit polarizers were replaced by the consortium in Oct, 2008, leading to improved stability, as expected. Thus, the observations done during our commissioning run were the one and only ever performed on AMBER without polarizers in the Medium-resolution spectral mode at $R=1500$.

Of the calibrators used, $\sigma$ Puppis stood out nicely, because the closure phase on this star varied from 0 to $180^{\circ}$ in a few hours: $\sigma$ Puppis, being a bright calibrator was also a large angular-size star and was completely resolved by our $\sim 90 \mathrm{~m}$ longest baseline, as shown in Fig. 1. However, the shallow slope of the closure phase transition from 0 to $\pi$ radians as the baseline crosses the first null needed an explanation. Part of the explanation was

\footnotetext{
Further author information:

G.D.: E-mail: gilles.duvert@obs.ujf-grenoble.fr, Telephone: +33 476514588

Optical and Infrared Interferometry II, edited by William C. Danchi, Françoise Delplancke, Jayadev K. Rajagopal, Proc. of SPIE Vol. 7734, 77340U · C 2010 SPIE · CCC code: 0277-786X/10/\$18 · doi: 10.1117/12.857970
} 


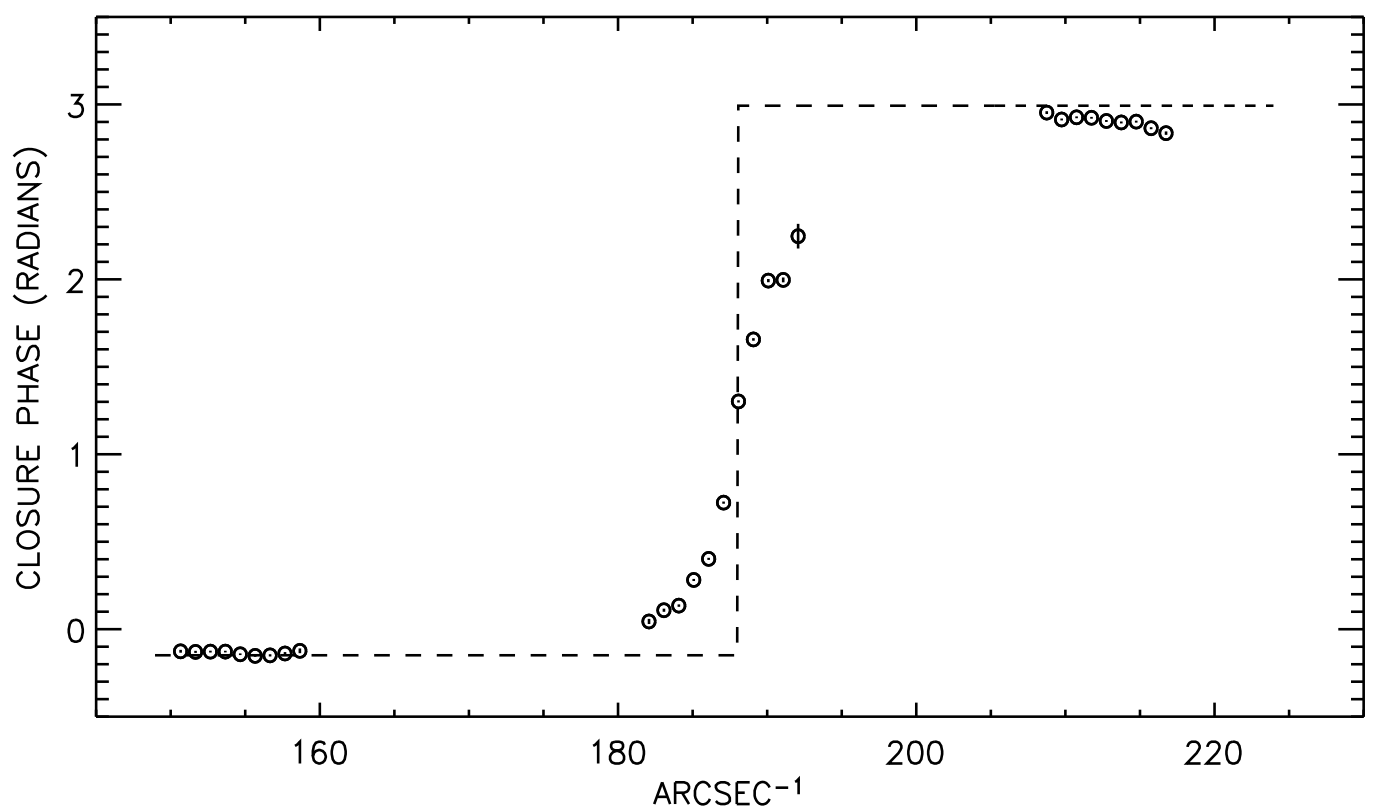

Figure 1. The uncalibrated closure phase of $\sigma$ Puppis (in radians) as a function of spatial frequency. Each of three groups of points corresponds to one five minute observation with VLTI/AMBER at $R=1500$ in $K$. Thanks to the spectral bandwidth used, each observation spans a range of spatial frequencies, so the transition from 0 to $\pi$ radians, as the baseline crosses the first null of the stellar disk Bessel function, is relatively well sampled. The data was binned to 1 arcsec ${ }^{-1}$ and the error bars, smaller than the symbols, are plotted. The overall shape is very different from the Heaviside jump expected from a stellar disk, with a smooth transition from 0 to $\pi$. Explaining this transition led us to develop the theory of Phase Closure Nulling.

quickly found in the literature: $\sigma$ Puppis was since 1907 known as a spectroscopic binary and should never have been included in a calibrator list. Thus, a probable reason for the deviation from a uniform stellar disk was the presence of the companion. However, $\sigma$ Puppis being of the SB1 type, the companion must be quite faint. The hypothesis that faint companions could have a visible imprint on the closure phase near the null led us to the development of the phase closure nulling concept.

\section{PHASE CLOSURE NULLING: THEORY}

An important property of stars resolved by long baseline interferometry is that the coherence of the light decreases with increasing spatial frequencies down to zero before increasing again following the well known behavior of the Bessel functions. Michelson and Pease ${ }^{3}$ used this property to measure the diameter of Betelgeuse for the first time.

\subsection{The uniform disk case}

For a star represented by an uniform disk of radius $R_{\star}$, the visibility is proportional to a Bessel function of the first order, that is

$$
V_{\star}(u)=2 \frac{J_{1}\left(2 \pi u R_{\star}\right)}{2 \pi u R_{\star}} .
$$

As shown in Fig. 2, $V_{\star}(u)$ is zero and the correlated flux of the star is canceled out at spatial frequencies multiples of $0.61 / R_{\star}$. Another property is that the phase of the visibility jumps by 180 degrees at each crossing of the Bessel function zeros (hereafter called visibility nulls or simply nulls). 

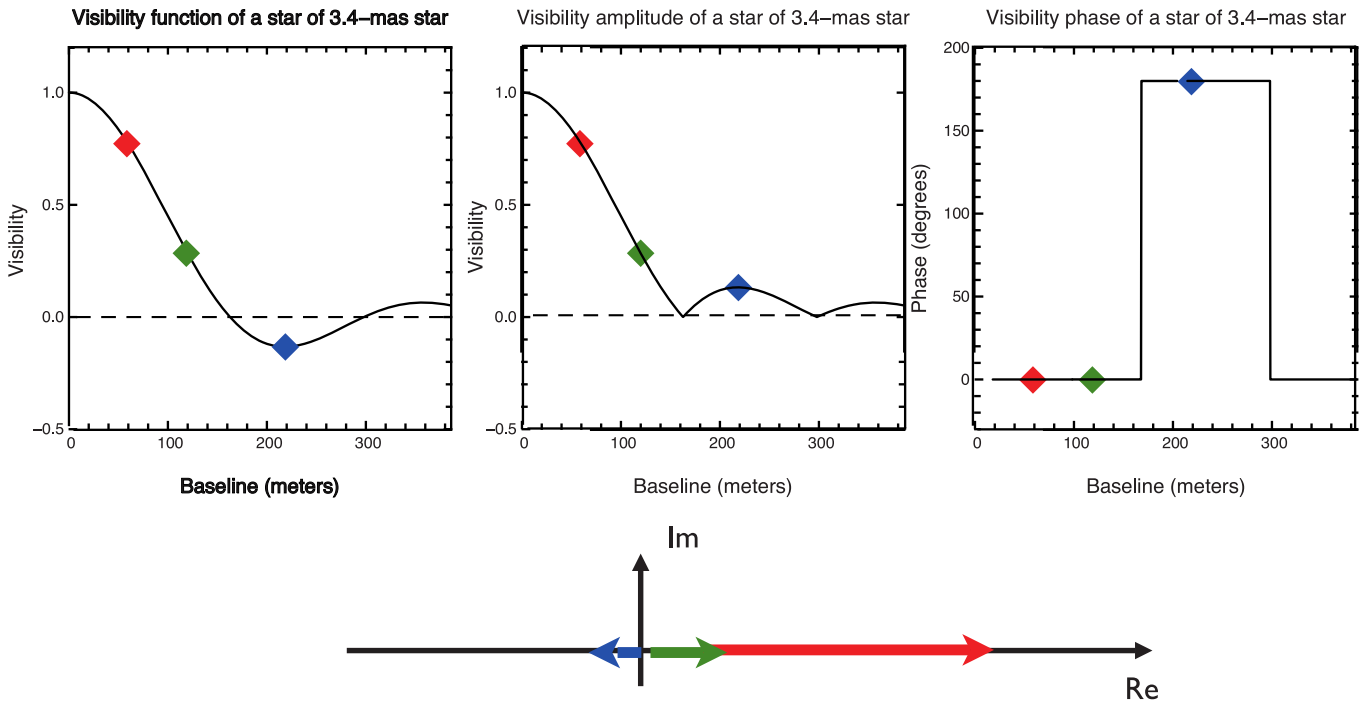

Figure 2. Sketch of the visibity function for a single star, showing the origin of the $\pi$ phase shift when one crosses a null. Left: complex visibility; middle: visibility amplitude; right: phase. Three points, selected at short, middle and high spatial frequencies, are represented as colored lozenges. The three corresponding complex visibilities are pictured at the bottom as Fresnel vectors in an Argand diagram showing the complex plane.

\subsection{Perturbation by a close faint companion}

If one adds a faint unresolved companion to the previous star, assuming for simplicity that the system orientation is parallel to the frequency axis, the visibility becomes

$$
\hat{\imath}(u)=\frac{V_{\star}(u)+r e^{i 2 \pi u s}}{1+r},
$$

where $r$ is the flux ratio and $s$ is the separation between the two components. For small flux ratios $r$, the amplitude of the visibility is only slightly modified by the presence of the companion. The stronger effect occurs around visibility nulls of the primary where the visibility perturbation is of the order of $r$. This effect remains weak and is beyond the performances of current interferometers as soon as the flux ratio is smaller than $1 \%$.

More interesting is the phase of the object visibility. The tangent of the phase of the previous system is given by

$$
\tan \phi(u)=\frac{r \sin (2 \pi u s)}{V_{\star}(u)+r \cos (2 \pi u s)} .
$$

As exemplified in Fig. 3, the phase is the result of two contributions: from the star $V_{\star}(u)$ and from the companion $r e^{2 i \pi u s}$. Except around visibility nulls of the primary, that is most of the time, the companion produces a phase signature in the range $\pm r / V_{\star}(u)$, which remains small. However, around visibility nulls, in the frequency ranges for which $\left|V_{\star}(u)\right| \leq r$, the phase signature of the companion becomes significant, with an exact value of $2 \pi u_{0} s$ at the frequencies $u_{0}$ of the nulls $\left(\approx \pi s / R_{\star}\right.$ for the first null), much greater than 180 degrees. It follows that, as opposed to the visibility amplitude, even for small flux ratios, there is always a frequency interval around nulls within which the phase signature of the companion is larger that any systematic error and is thus measurable.

\subsection{The phase closure properties}

Unfortunately, the absolute phase of an interferogram is a quantity difficult to measure as it requires an absolute reference that in general does not exist. But, with 3 or more telescopes, one can use instead the closure phase $\phi_{c}$ defined as the phase of the bispectrum on 3 baselines $\hat{I}\left(u_{12}, u_{23}\right)$, with

$$
\hat{I}\left(u_{12}, u_{23}\right)=<\hat{\imath}\left(u_{12}\right) \hat{\imath}\left(u_{23}\right) \hat{\imath}^{*}\left(u_{13}\right)>,
$$



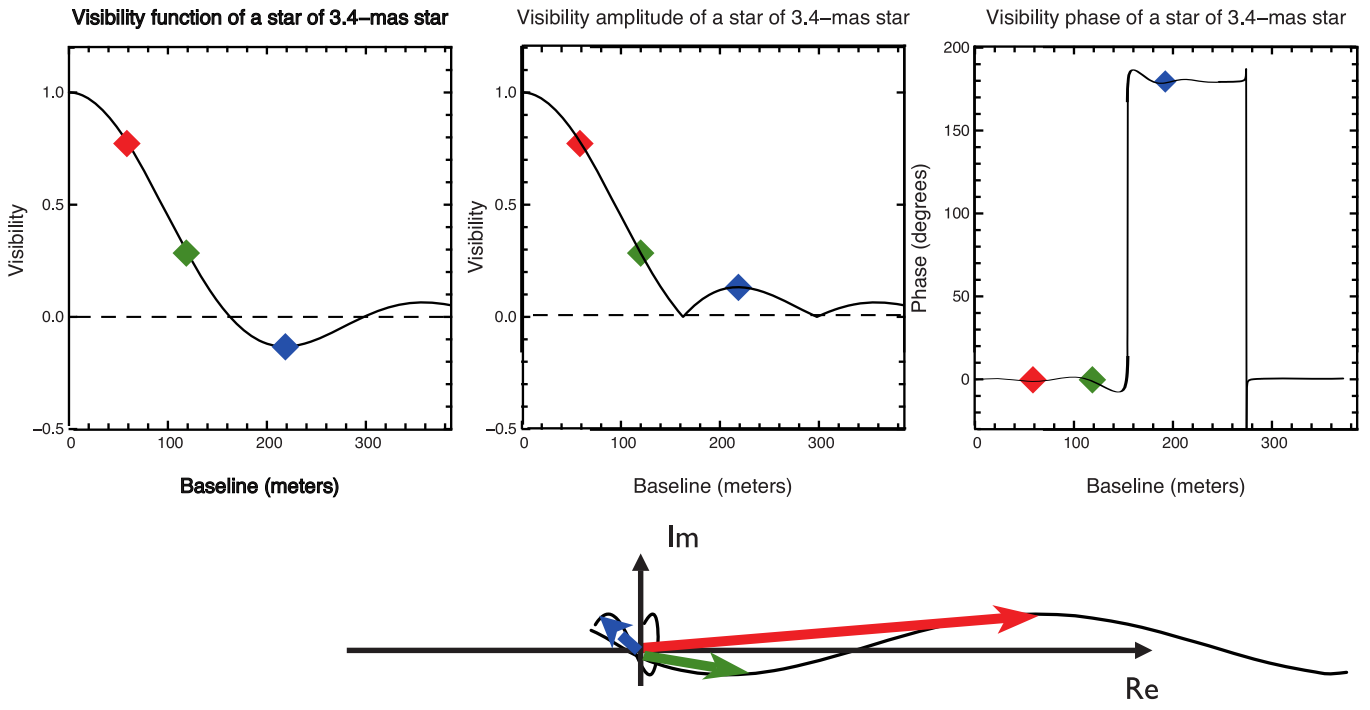

Figure 3. Sketch of the visibility function for a star plus faint companion, with the same layout as Fig. 2. The bottom Argand diagram displays the corresponding Fresnel vectors. The imaginary part due to the faint companion induces a phase of the visibility that can become quite large near the visibility null at the origin.

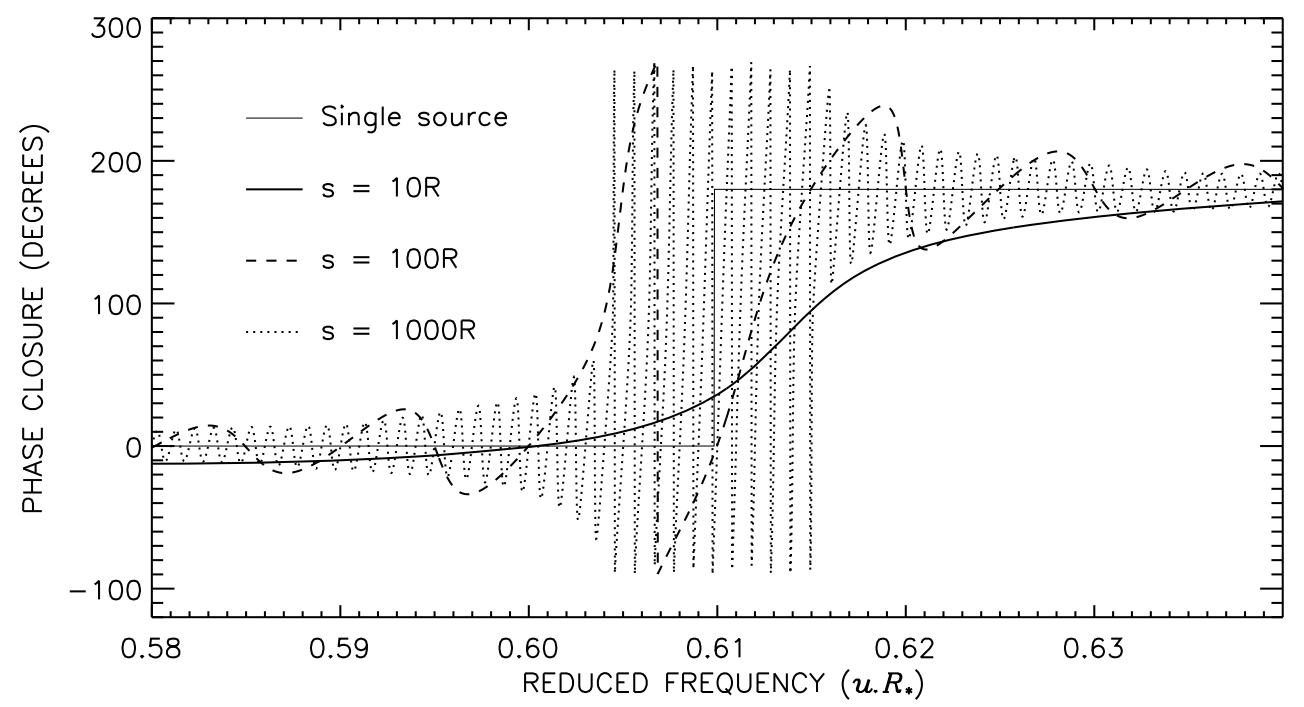

Figure 4. Closure phase modulo $2 \pi$ of a double system, formed by an extended uniform disk and a point source, as a function of the maximum reduced frequency. The spectral resolution is $\mathcal{R}=1500$. The 3 frequencies $\left\{u_{23}, u_{12}, u_{13}\right\}$ (see Eq. 4) have been chosen in the ratio 1,2,3, with the maximum frequency around the first zero of visibility of the primary star. The closure phase is displayed for 3 separations $\left(10 R_{\star}, 100 R_{\star}\right.$, and $\left.1000 R_{\star}\right)$ and a flux ratio of 0.01 . The signature of the secondary source is dominant in the region around the minimum visibility of the primary (near reduced frequency 0.61). The thin line with a 180 degree shift at 0.61 corresponds to the closure phase of the primary alone.

where $u_{12}, u_{23}$ and $u_{13}$ are the 3 frequencies transmitted by the interferometer with $u_{13}=u_{12}+u_{23},{ }^{*}$ denotes the complex conjugate, and $\langle>$ represents an ensemble average. The closure phase is a self calibrated observable that unlike the phase does not need an absolute reference. In addition, it coincides to the closure phase of the observed object, that is

$$
\phi_{c}=\phi_{o}\left(u_{12}\right)+\phi_{o}\left(u_{23}\right)-\phi_{o}\left(u_{13}\right),
$$

where $\phi_{o}$ is the phase of the object spatial Fourier transform.

Fig. 4 shows the closure phase around the first visibility null of the primary, from a double system formed by 


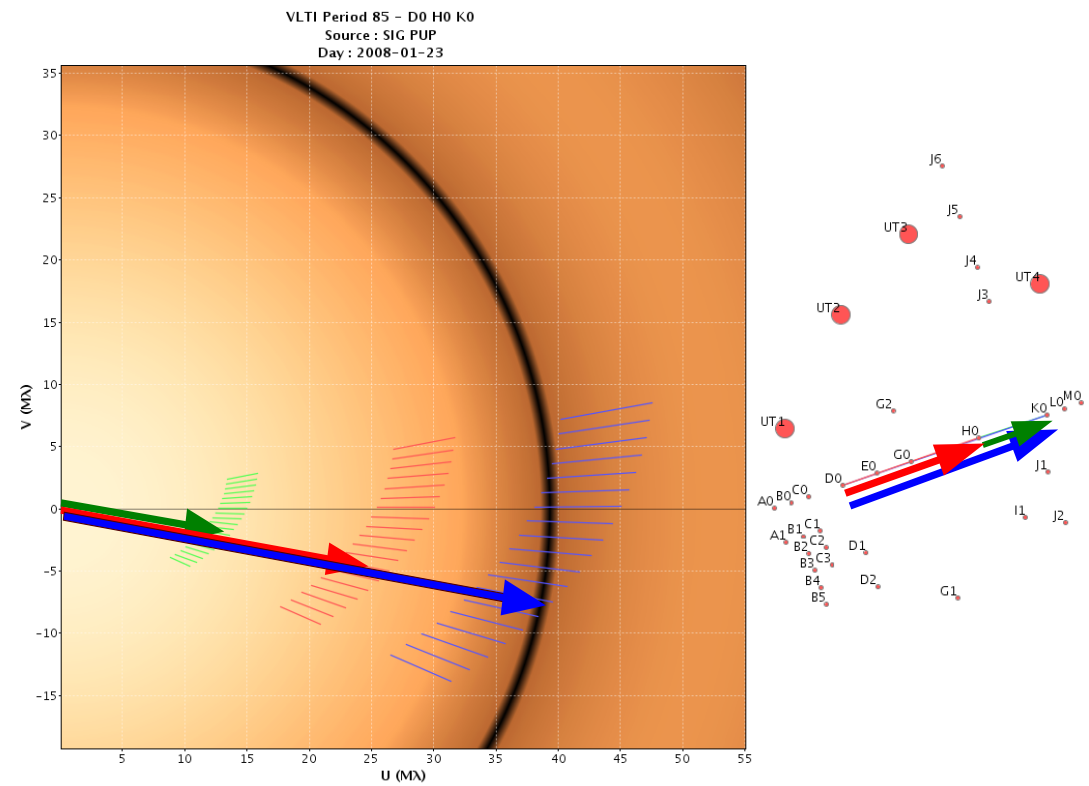

Figure 5. Sketch of the setup used at VLTI for PCN observations. The right panel shows the stations of the VLTI and the D0-H0-K0 triplet used. The left panel shows the $(u, v)$ plane and a 2-dimension map of the amplitude of the Fourier transform of a star of 6.4 mas ( $\sigma$ Puppis). The green, red and blue lines correspond to the baselines H0-K0, D0-H0 and D0-K0 respectively, the latter sweeping above the first null (black circle) of the star due to the combination of the supersynthesis effect and the spatial resolution spanned by the observational bandwidth.

an extended uniform disk and an unresolved companion with a flux ratio of $1 \%$ at various separations. Note the importance of the closure phase signature from the companion. PCN is all about observing closure phases near such nulls, where the correlated flux of the star is vanishing, and fitting in the closure phase shape a stellar disk and companion, that is, three basic parameters, $R_{*}, r$ and $s$. We note that, as shown in Fig. 5, in various observational cases with two short baselines and a long one, the closure phase equals the phase of the long baseline since the phases on the two short baselines can be approximated by that of an unresolved stellar disk, i.e., zero.

\subsection{Performances}

The performances of PCN have been analyzed in Chelli et al. (2009). ${ }^{1}$ We summarize here the main properties:

- $\phi_{c}$ being proportional to $r / V_{*}$, there is no gain in SNR in the null. One still have to integrate enough to get the signal out of photon and detector noises.

- Fibered interferometers have a field of view of one Airy disk. The field of view combined to the necessity to resolve the parent star imposes a maximum recoverable separation of $s_{\max } / R_{*} \approx B / D$, which translates to a maximum of $\sim 100 R_{*}$ for VLTI.

- In the photon noise regime, the error on the flux ratio is given by $\epsilon(r) \approx 3 / S \sqrt{K}$ ( $S$ being the Strehl ratio and $K$ the total number of photons), which is 3 times worse than direct detection.

\subsection{Proof of concept: PCN on $\sigma$ Puppis}

Although sampling quite sparsely the transition region around the first null, the $\sigma$ Puppis observations depicted in Figs. 1 and 5 have been interpreted in terms of PCN by Duvert et al. (2010). ${ }^{5}$ The author best fit was $R_{*}=3.23 \pm 0.015$ mas, a magnitude difference of $5.3 \pm 0.2$ between the giant and its companion, and a projected distance at time of observation of 11 mas, that is, 4 stellar radii. With these numbers, assuming that the companion is a main-sequence star, they were able to estimate in two independent ways the masses in the system, both estimate giving the same result: a $5 M_{\odot} \mathrm{K} 5 \mathrm{III}$ primary and a $\mathrm{A} 2 \mathrm{~V} 2.2 M_{\odot}$ secondary. 


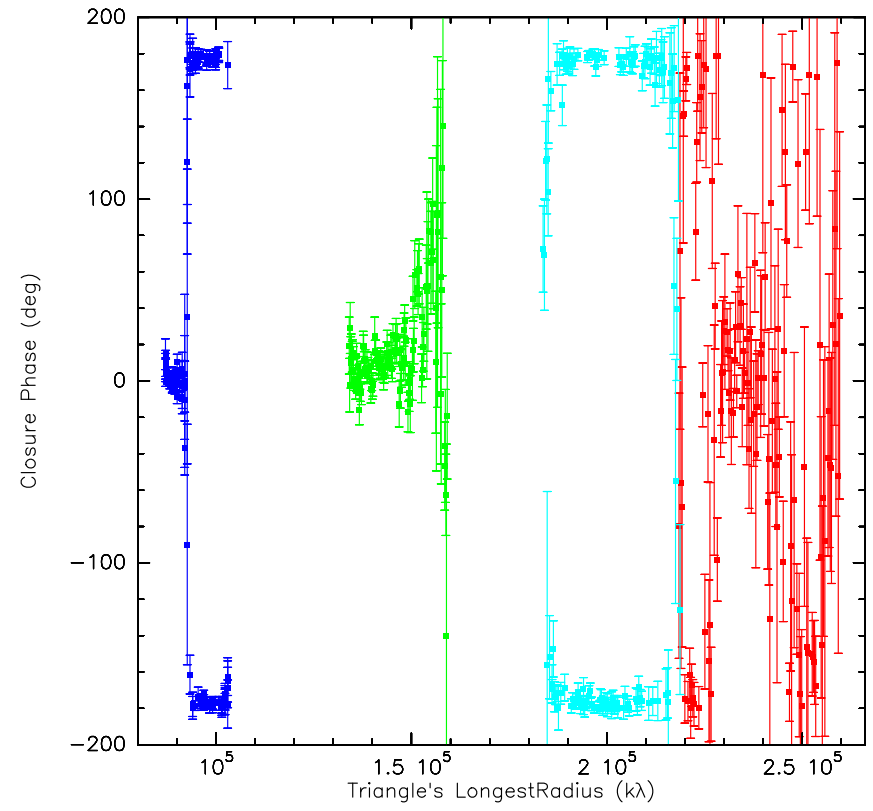

Figure 6. CHARA/MIRC observation of the star $\iota$ Dra in July 2009, with several crossings of the null with closure phase jumps. The plot groups the phase closures on the 4 triplets in all the 24 channels of MIRC/GRISM (H band), as a function of the spatial frequency of the longest baseline in the triplet. From left to right: in blue, the triplet E1-E2-W2; in green, the E1-E2-W1; in light blue, E2-W1-W2; and in red, E1-W1-W2.

\section{SCIENCE CASES}

There are a number of science cases that can benefit from the possibility to detect, to very high dynamics, the presence of a faint companion near a bright star, i.e., inside the airy disk patch of light that a star produces in a conventional telescope:

- PCN can be complementary of spectroscopic diagnostics of binarity, in the cases when the inclination or period of the double system become unfavourable ${ }^{4}$ for an example of limitations of radial velocity detection of planetary companions).

- PCN can perform the spectroscopy of the companion, when the $\mathrm{S} / \mathrm{N}$ per channel is sufficient, the companion spectrum being the stellar spectrum multiplied by the flux ratio $\mathrm{r}$ estimated for each spectral channel.

- For already know spectroscopic binaries of type SB1 with a giant component that can be spatially resolved by the interferometer, a single PCN detection can give the nature of the companion, and subsequently the mass of the giant star. The details of the method used are to be found in Duvert et al. (2010). ${ }^{5}$

- PCN can help in finding Brown dwarves companions, and in general extend binarity statistics towards the low mass ratio limit.

- finally, in favourable cases, one could expect to detect Hot Jupiters and at least measure their orbit and inclination.

A rough estimate for the number of targets feasible with the VLTI are of $\approx 100$ stars in $K$ and $\approx 500$ in J.

\section{OBSERVATIONAL CAMPAIGN 2009}

We started an observational campaign in 2009 on two interferometric facilities: AMBER/VLTI ${ }^{6}$ with 3 ATs, in Medium-Resolution $K(R=1500)$, for which we got 11 nights, and, MIRC/CHARA, ${ }^{7}$ in $K, R=400$, for which we got 1 useful observing night (see Fig. 6). 

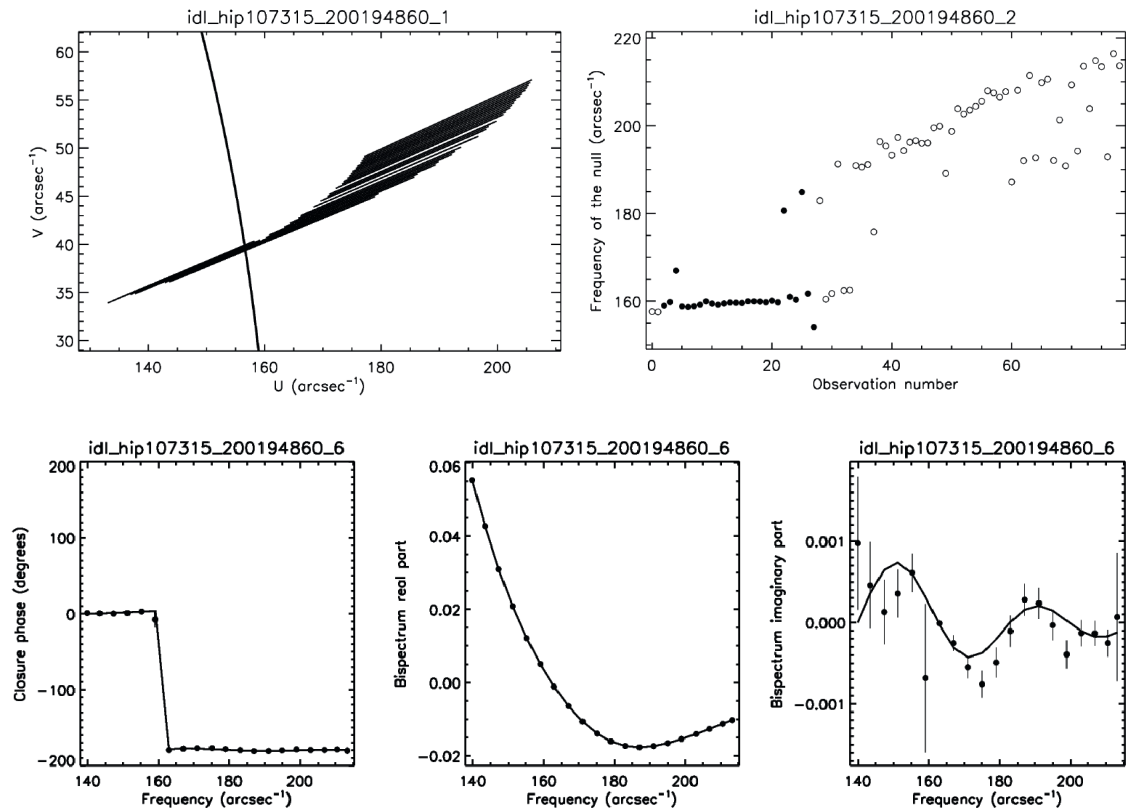

Figure 7. First results of PCN on HIP 107315. See text for details.

In the rest of the section, we focus on the AMBER/VLTI data. The targets were of two types: SB1 stars with a giant primary, and, giant stars for which no companion is known, which could serve either as a mean to test the sensitivity limit, or eventually, would actually possess a faint companion.

\subsection{Observational procedure}

The observational procedure was very simple. We got an estimate of the star diameter using the SearchCal ${ }^{8}$ web service. Given the conservative uncertainties on the stellar diameters obtained this way, the time of crossing of the first null was not very precisely known. So we tried to stay as long as possible on the science star, typically 4 to 5 hours, bracketing the observations with a $1 \mathrm{~h}$ observation of two calibrators, usually smaller, thus fainter objects, giving much noisier measurements. Normally for closure phase, a calibration is not really necessary. However in the case of AMBER, the closure phase obtained on a star will contain the phase closure of the so-called P2VM, the calibration matrix used to estimate the coherent fluxes. It is thus necessary to correct this closure phase from the one of a calibrator.

For the data reduction we used the new amdlib3 ${ }^{9}$ algorithm which provides accurate complex coherent fluxes.

We did not use the VLTI fringe tracker since it operates at $H$ band, and, as can be seen in Fig. 5, if in $K$ the stars are fully resolved at the distance of the longest baselines, in $H$ they are already resolved at the distance of the second longest baseline, and the small coherent flux at that point prevent a reliable fringe correction: fringe trackers should operate at a longer wavelength than the science observation!

\subsection{Preliminary observations}

We focused firstly on the AMBER/VLTI data since we had previous experience of such data with our $\sigma$ Puppis observations. Regrettably, AMBER observations in 2009 suffer from an important closure phase noise, typically $5^{\circ} \mathrm{rms}$. This comes from both an integration time per frame $(\approx 100 \mathrm{~ms})$ that is quite long with respect to the coherence time at $K$, and from a piston-phase dependency induced by a dichroic in the VLTI beams, optical element that has been replaced in November 2009. So, for this campaign, we cannot achieve the ultimate in sensitivity possible with AMBER/VLTI, and were obliged to develop new techniques to reduce the data.

We have at the moment run only a general analysis on most of the single stars in our observation list. This analysis consists in a two-pass method. We first find the position of the zero of the real part of the bispectrum, to get a first good estimate of the stellar diameter. Then, we use this as an initial value to fit $R_{*}, s$ and $r$. 

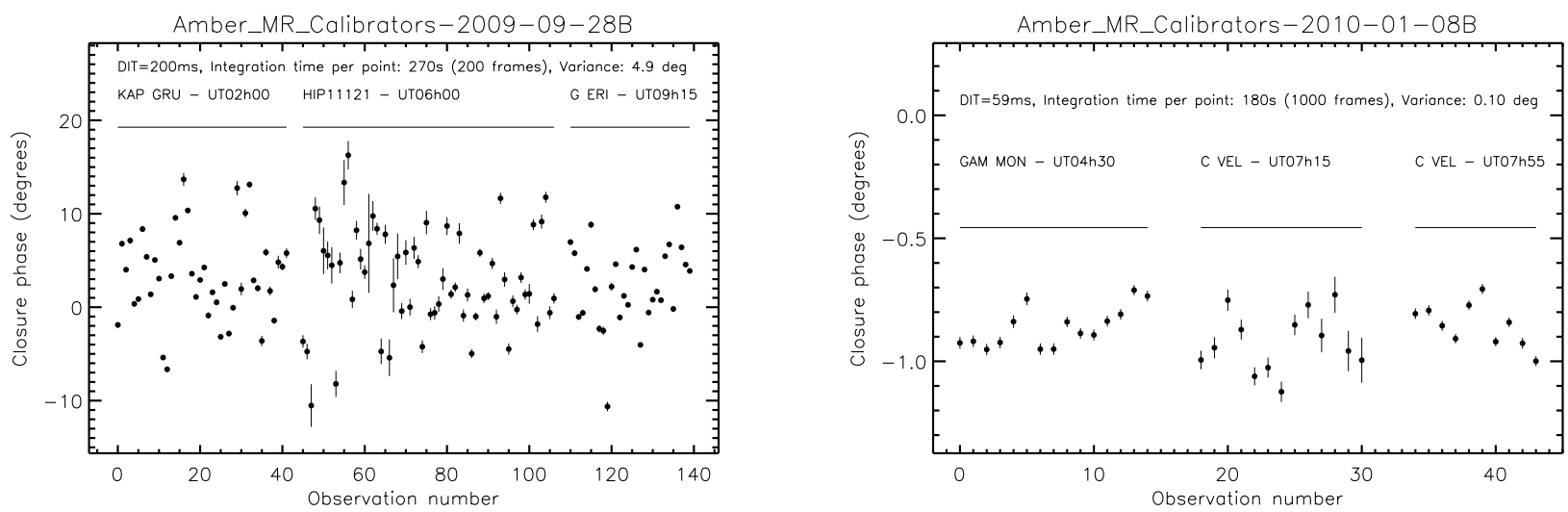

Figure 8. Improved stability of the closure phase of AMBER from 2009 to 2010. Plots of the closure phase as a function of time for calibrators of different fluxes and at very different elevation in 2009 (left) and in 2010 (right) in respectively $7 \mathrm{~h}$ and $3 \mathrm{~h}$ spans. The stability of AMBER has improved by a factor 50 from a variance of $4.9^{\circ}$ in 2009 to a variance of $0.1^{\circ}$ in 2010.

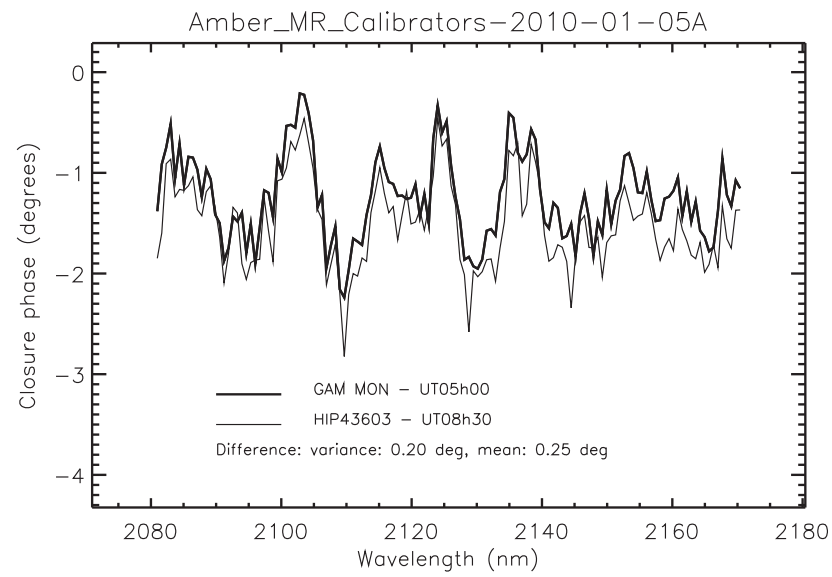

Figure 9. Improved stability of the closure phase of AMBER in 2010: plot of the closure phase as a function of wavelength for 2 calibrators, of different fluxes and at very different elevation, taken at 4 hours intervals. The variance is $0.2^{\circ}$.

Figure 7 show the two main aspects of the process for one of our objects, HIP 107315. In panel (a), we show the $(u, v)$ coverage of the observations with the approximate position of the null. One sees that in this case our observations cut the null at a constant angle. The right (b) panel show the result of a the fit of $R_{*}$, which gives extremely precise values for the star diameter in all observations where the null is crossed (filled circles). From these observations we derive $R_{*}=3.81875 \pm 0.018$ mas. In the lower three panels, we plot, from left to right, the shape of the closure phase (c), the real part of the bispectrum (d) and the imaginary part (e), where a sine-like signal of very small amplitude can be seen. If this signal comes indeed from a companion (physically linked or field star), the separation is not well constrained yet by the observations, but the flux ratio can be reliably estimated to be $3.310^{-3}$. Thus, with the 2009 observations that suffer from a large closure phase error, we can achieve already detections of faint companions.

\subsection{Update on the AMBER closure phase stability}

The stability of the closure phase of AMBER has improved dramatically since the replacement of the IRIS dichroic in the last months of 2009. We made new closure phase observations in early 2010, and, as shown in Fig. 8, the closure phase does not vary from more than $0.1^{\circ}$ in a few hours (instead of $5^{\circ}$ in our 2009 observations). This is also visible in the spectral direction, as seen in Fig. 9, where the fixed phase artifacts are stable and time and can be calibrated down to $0.2^{\circ}$ at least.

Although not fully understood, the closure phase dependence on the piston induced by the previous IRIS dichroic 
was certainly related to differential polarization effects. In this respect, it is remarkable that our discovery of the PCN effect arose from the sole unpolarized observations made with AMBER.

\section{CONCLUSION AND PERSPECTIVES}

Phase Closure Nulling is an observational technique consisting in measuring closure phases of resolved objects with a baseline triplet where one of the baselines crosses a null of the object visibility function. In these regions the signature of fainter scene objects are seen in the closure phase, and stay out since the correlated flux of the star is essentially nulled at these positions. PCN is limited in field of view and needs quite high spectral resolution, but can achieve detection of high-contrast objects. We report a first estimate of contrasts down to $10^{-3}$ obtained in 2009 on AMBER with a bad closure phase rms of $\approx 5^{\circ}$. We think that the detection limit can be pushed much farther thanks to the the excellent closure phase stability $\left(\sim 0.1^{\circ}\right)$ displayed by AMBER since

a hardware change in VLTI that took place in November 2009.

\section{ACKNOWLEDGMENTS}

The authors warmly thank the ESO/VLTI and CHARA teams for their support during the observations.

\section{REFERENCES}

[1] Chelli, A., Duvert, G., Malbet, F., and Kern, P., "Phase closure nulling. Application to the spectroscopy of faint companions," A\&A 498, 321-327 (2009).

[2] Malbet, F., Duvert, G., Kern, P., and Chelli, A., "February 2008 AMBER Task Force run report," tech. rep., ESO Doc No. VLT-TRE-AMB-15830-7120, issue 1.2, dated 16/04/2008 (arXiv: 0808.1315) (2008).

[3] Michelson, A. A. and Pease, F. G., "Measurement of the diameter of alpha Orionis with the interferometer.," ApJ 53, 249-259 (1921).

[4] Meunier, N., Desort, M., and Lagrange, A., "Using the Sun to estimate Earth-like planets detection capabilities . II. Impact of plages," $A \mathscr{E} A \mathbf{5 1 2}, \mathrm{A} 39+(2010)$.

[5] Duvert, G., Chelli, A., Malbet, F., and Kern, P., "Phase closure nulling of HD 59717 with AMBER/VLTI . Detection of the close faint companion," $A \& A$ 509, A66+ (2010).

[6] Petrov, R. G., Malbet, F., Weigelt, G., and coll., "AMBER, the near-infrared spectro-interferometric threetelescope VLTI instrument," A\& A 464, 1-12 (2007).

[7] Monnier, J. D., Pedretti, E., Thureau, N., Berger, J.-P., Millan-Gabet, R., ten Brummelaar, T., McAlister, H., Sturmann, J., Sturmann, L., Muirhead, P., Tannirkulam, A., Webster, S., and Zhao, M., "Michigan Infrared Combiner (MIRC): commissioning results at the CHARA Array," in [Society of Photo-Optical Instrumentation Engineers (SPIE) Conference Series], Presented at the Society of Photo-Optical Instrumentation Engineers (SPIE) Conference $\mathbf{6 2 6 8}$ (2006).

[8] Bonneau, D., Clausse, J., Delfosse, X., Mourard, D., Cetre, S., Chelli, A., Cruzalèbes, P., Duvert, G., and Zins, G., "SearchCal: a virtual observatory tool for searching calibrators in optical long baseline interferometry. I. The bright object case," A\&A 456, 789-789 (2006).

[9] Chelli, A., Utrera, O. H., and Duvert, G., "Optimised data reduction for the AMBER/VLTI instrument," $A \& 3 A$ 502, 705-709 (2009). 